\title{
IVF treatment of moderate male factor infertility: a comparison of mini-Percoll, partial zona dissection and sub-zonal sperm insertion techniques
}

\author{
D.Sakkas ${ }^{1}$, L.Gianaroli², L.Diotallevi², I.Wagner, \\ A.P.Ferraretti ${ }^{2}$ and A.Campana \\ Clinique de Stérilité et d'Endocrinologie Gynécologique, Hôpital \\ Cantonal Universitaire de Genève, 32 Boulevard de la Cluse, \\ 1211 Geneva 14, Switzerland and ${ }^{2}$ IVF Centre, Casa di Cura, \\ Villa Regina, via Castiglione, Bologna, Italy \\ 'To whom correspondence should be addressed
}

In this study we examined various techniques of in-vitro fertilization (IVF) for treating couples in whom the male had subnormal semen parameters. We compared two sperm preparation methods (mini-Percoll and conventional swimup) for efficiency of recovery after preparation and for fertilization rates after IVF, and compared the suitability of partial zona dissection (PZD) and sub-zonal sperm insertion (SUZI) to patients with different types of male factor infertility. The mini-Percoll technique allowed the recovery of significantly more motile spermatozoa from the same semen sample compared to the swim-up method. More oocytes were fertilized after spermatozoa were prepared by the mini-Percoll technique. An increased number of spermatozoa recovered from an ejaculate led to an improvement in the quality of spermatozoa in the insemination droplet. Subsequently, when using the PZD technique, the fertilization rate increased when there was a higher number of spermatozoa in the patient's ejaculate. When comparing the two micromanipulation techniques, SUZI provided patients with oligoasthenzoospermia (i.e. $<10 \times 10^{6}$ spermatozoa $/ \mathrm{ml}$ and $40 \%$ motility) with a higher chance of obtaining 2-pronculeate eggs.

Key words: male infertility/partial zona dissection/sperm preparation/sub-zonal sperm insertion

\section{Introduction}

The treatment of subfertile males by routine in-vitro fertilization (IVF) is limited in many cases by the low number of normal spermatozoa that can be harvested from an ejaculate, or because of an inability of the spermatozoa to bind or pass through the zona pellucida or oocyte membrane (Overstreet et al., 1980; Cohen et al., 1985). In these cases, the chances of fertilization can be improved either by attempting to harvest more spermatozoa for insemination by methods such as the mini-Percoll technique (Ord et al., 1990), or by the use of micromanipulation techniques which increase the accessibility of the oocyte to the spermatozoon.

The main micromanipulation methods currently in clinical use are sub-zonal sperm insertion (SUZI), which requires the (c) Oxford University Press insertion of one or more spermatozoa into the perivitelline space, or partial zona dissection (PZD), which creates a slit-like incision in the zona pellucida. Both techniques have been successfully adopted by several groups and a number of pregnancies have now been reported ( $\mathrm{Ng}$ et al., 1988; Cohen et al., 1989; Malter and Cohen, 1989; Fishel et al., 1990; Palermo et al., 1992; Sakkas et al., 1992). The mini-Percoll technique has been applied to less severe cases of male factor infertility and has also been successful (Ord et al., 1990).

The availability of these methods has led to the necessity of selection criteria for males who could be treated either by routine IVF procedures or by SUZI and/or PZD. In addition, due to the variability in male factor cases, certain patients may be better suited to treatment by normal IVF techniques or PZD as compared to SUZI.

The present study compares the suitability of some of these techniques (mini-Percoll, PZD and SUZI) to a range of moderate male factor patients in order to assess which techniques are more applicable to certain types of male factor infertility.

\section{Materials and methods}

\section{Patient selection}

The study involved couples in which the males exhibited oligozoospermia, asthenozoospermia or teratozoospermia alone or combinations of these indications according to the World Health Organization (WHO, 1987) guidelines. The patients in this study were undergoing IVF treatment at the Clinique de Stérilité et d'Endocrinologie Gynécologique, Hôpital Cantonal Universitaire de Genève, Geneva, Switzerland, and the Casa di Cura, Villa Regina, Bologna, Italy.

\section{Oocyte collection}

Oocytes were aspirated from ovarian follicles shortly before the estimated time of ovulation following the stimulation protocol previously described by Gianaroli et al. (1986). The oocytecumulus complex was cultured in Whittingham's T6 medium (Quinn et al., 1982) supplemented with $10 \%$ human serum (HS) at $37^{\circ} \mathrm{C}$ in an atmosphere of $5 \% \mathrm{CO}_{2}$ in air. For the micromanipulation procedures, the cumulus-oophorus and corona radiata were removed with hyaluronidase $(3 \mathrm{IU} / \mathrm{ml}$; Leech Type X, Sigma Pharmaceuticals, Buchs, Switzerland), dissolved in HEPES buffered T6 medium, and by gentle aspiration through a finely drawn glass pipette.

\section{Semen preparation: mini-Percoll and swim-up methods}

To compare the conventional swim-up and mini-Percoll techniques, aliquots were taken from the same semen sample and 
a comparison made of sperm recovery after both preparation techniques. This comparison involved 28 males who had presented for treatment of infertility. Semen was prepared using T6 medium containing $10 \% \mathrm{HS}$. The mini-Percoll technique was performed as described by Ord et al. (1990).

A number of patients who had entered the Geneva Clinic for an IVF treatment cycle, prior to the availability of micromanipulation techniques, were also compared for fertilization rates obtained after sperm preparation by these techniques. In these couples the males possessed sub-normozoospermic semen parameters (Table I). Spermatozoa were prepared by either mini-Percoll or swim-up and oocytes were inseminated by $50000-70000$ motile spermatozoa per $\mathrm{ml}$ in a Nunc well containing $0.4 \mathrm{ml}$ total volume.

\section{Micromanipulation: partial zona dissection (PZD) and sub-zonal sperm micro-injection (SUZI)}

This part of the study included 25 male factor patients who were considered unsuitable for treatment using routine IVF insemination techniques because of poor semen parameters or repetitive failure of IVF. In the course of this study, patients with severe male factor infertility were treated by SUZI alone because not enough spermatozoa were obtained to form an insemination droplet. After ejaculation the semen sample was incubated for 20 min with $3 \mathrm{mM}$ 2-deoxyadenosine (Sigma Pharmaceuticals) at $37^{\circ} \mathrm{C}$ and then prepared using the mini-Percoll procedure.

Once treated with hyaluronidase, oocytes judged to be in the MII phase were randomly allocated to the SUZI and PZD groups in a 60:40 ratio for each patient. In six cases, patients had $>16$ oocytes collected and a small number of eggs were also allocated for insemination by routine IVF procedure. Of these six cases, four had $>20 \times 10^{6}$ spermatozoa/ml but $<20 \%$ motility and had previously failed to achieve fertilization in one or two IVF cycles. The other two cases were oligoasthenozoospermic but had enough spermatozoa to perform the three techniques concurrently. Patients with $<16$ oocytes were not allocated to all three techniques in order not to jeopardize their chances of fertilization.

The SUZI procedure was performed as previously described (Sakkas et al., 1992). In all cases, between four and six spermatozoa were placed into the perivitelline space. The PZD procedure was performed as described by Malter and Cohen (1989). Once the eggs had their zona pierced by the PZD procedure, they were pooled and combined with spermatozoa

Table I. Comparison of sperm recovery and fertilization rates of male factor patients presenting for infertility treatment prior to availability of micromanipulation techniques, after sperm preparation by mini-Percoll or conventional swim-up methods

\begin{tabular}{lcc}
\hline & Mini-Percoll & Swim-up \\
\hline No. of patients & 23 & 7 \\
Initial sperm concentration $\times 10^{6}$ & $7.4 \pm 5.8$ & $9.9 \pm 5.3$ \\
$\quad($ mean \pm SD) & $32.3 \pm 13.4$ & $49.0 \pm 11.9$ \\
Initial sperm motility, \% (mean \pm SD) & $3.2 \pm 2.8$ & $1.5 \pm 1.3$ \\
Final sperm concentration $\times 10^{6}$ & & 36 \\
$\quad$ (mean \pm SD) & 138 & $0(0)$ \\
No. of eggs & $30(21.7)$ & \\
No. of eggs fertilized (\%) & & \\
\hline
\end{tabular}

at a concentration of $50000-70000$ motile spermatozoa $/ \mathrm{ml}$. The following morning, 16-20 $\mathrm{h}$ after micromanipulation, the eggs were assessed for the presence of two pronuclei (PN). Two PN observed later than $20 \mathrm{~h}$ after manipulation were regarded as delayed fertilizations and not included in the results. Once fertilized, the zygotes were transferred to the Fallopian tubes of the patient by tubal embryo stage transfer (TEST), or embryos were transferred directly to the uterus.

\section{Statistical analysis}

The analysis of sperm concentration recovered after treatment by mini-Percoll and swim-up was performed using Student's $t$-test. Comparison of the fertilization results achieved by the different procedures was performed using the $\chi^{2}$-test.

\section{Results}

\section{Mini-Percoll and swim-up methods}

The final concentration of spermatozoa recovered from aliquots of the same ejaculate was significantly higher after mini-Percoll preparation compared to that after swim-up (Figure 1). The percentage of motile spermatozoa in the final preparation was not significantly different; $70 \%$ after mini-Percoll compared to $80 \%$ after swim-up. Among the male factor patients from whom data were obtained prior to the availability of micromanipulation techniques, all seven who had spermatozoa prepared for an IVF treatment cycle by swim-up achieved no fertilization, while nine of the 23 patients who had spermatozoa prepared using the mini-Percoll technique achieved a mean fertilization rate of $21.7 \%$ (Table I).

\section{Semen characteristics of patients treated by $P Z D$ and SUZI}

Of the 25 patients examined, 16 exhibited some form of oligoasthenoteratozoospermia according to the WHO guidelines (WHO, 1987). The main difference between patients was the severity of oligozoospermia. On the day of treatment, nine patients had $<10 \times 10^{6}$ spermatozoa $/ \mathrm{ml}$ while a further $10 \mathrm{had}$ between 10 and $20 \times 10^{6}$ spermatozoa/ml. All but one patient had $<50 \%$ motility. Four of the patients with $>20 \times 10^{6}$

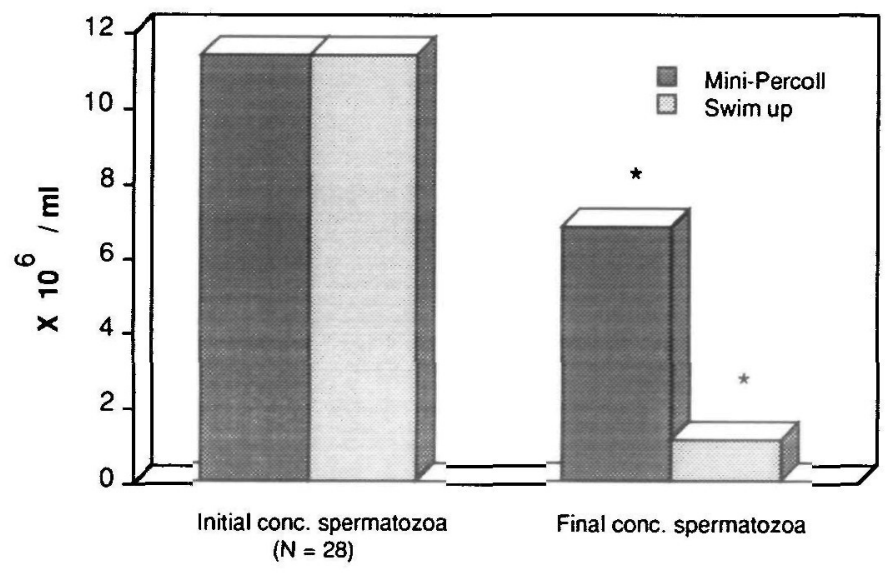

Fig. 1. The final concentration of motile spermatozoa recovered from aliquots of the same ejaculate after using the mini-Percoll and conventional swim-up sperm preparation techniques $(* P<0.01)$. 
spermatozoa/ml had failed to achieve fertilization in two previous cycles. Of the the 22 patients assessed for sperm morphology, 16 had $>50 \%$ abnormal forms in their semen sample. Three patients were not assessed for sperm morphology as there were insufficient spermatozoa after treatment.

\section{Comparison of PZD and SUZI}

Overall, the total number of eggs fertilized by the SUZI and PZD techniques was $46 / 190(24.2 \%)$ and $24 / 116(20.7 \%)$ respectively, while the number of two PN eggs obtained was $42 / 190$ (22.1\%) and $16 / 116(13.8 \%)$ respectively. Of the eggs fertilized, the number of polyspermic eggs after the PZD technique was $8 / 24$ $(33.3 \%)$, significantly higher $\left(\chi^{2}=5.12 ; P<0.05\right)$ than that after SUZI $(4 / 46 ; 8.7 \%)$. Continual assessment of the unfertilized eggs $20 \mathrm{~h}$ after micromanipulation indicated a further five fertilizations in the SUZI group and four in the PZD group; delayed fertilization was observed up to $48 \mathrm{~h}$ post-manipulation.

In investigating which patients were more likely to have fertilization with either SUZI or PZD it was observed that patients who had an initial sperm count of $<10 \times 10^{6}$ spermatozoa $/ \mathrm{ml}$ all achieved fertilization by the SUZI technique, while only two such patients were successful when using PZD. In this group of patients significantly more $\left(\chi^{2}=6.33 ; P<0.05\right)$ two PN eggs were obtained using SUZI (Figure 2). In contrast, of the 16 patients with an initial sperm count of $>10 \times 10^{6}$ spermatozoa/ml, 14 had fertilization using SUZI and 11 when using PZD. In this group of patients there was no significant difference in the number of two PN eggs obtained; however, more polyspermic eggs were observed after PZD treatment. In this study, a comparison of the fertilization rate according to motility and morphology was difficult as the patients selected for the study all had spermatozoa displaying similar motility and morphology parameters, while patients with severe asthenozoospermia (i.e. insufficient motile spermatozoa to perform PZD or IVF) were treated only by SUZI.

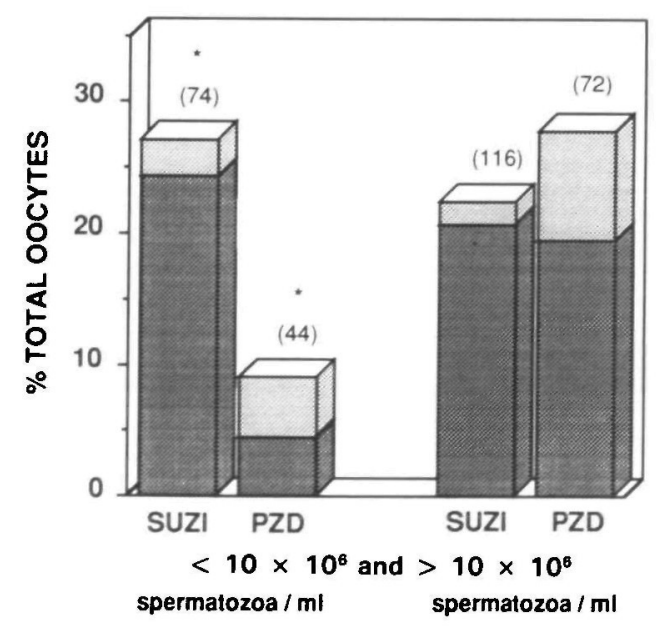

Fig. 2. Comparison of the percentage of two pronuclear (W) and polyspermic (咚) oocytes obtained after performing sub-zonal sperm microinjection (SUZI) or partial zona dissection (PZD) with semen samples containing less than $(n=9)$ or greater than $(n=16)$ $10 \times 10^{6}$ spermatozoa $/ \mathrm{ml}$. The number of oocytes treated is in parentheses.
In six cases PZD, SUZI and IVF were all performed on oocytes from the same patient. A statistically significant difference $(P<0.05)$ was found in the number of eggs fertilized after both SUZI and PZD compared to IVF (Figure 3).

\section{Embryo transfer}

None of the nine patients receiving embryo transfer after mini-Percoll preparation and routine IVF became pregnant. Of the 25 patients who had fertilized eggs transferred after treatment by SUZI and/or PZD, one became pregnant after a uterine transfer of three PZD embryos (two 4-cell embryos and one 2-cell embryo) and a pronuclear stage microinjected zygote which was still displaying its pronuclei $41 \mathrm{~h}$ post-injection. The male partner in this case had a sperm count of $15 \times 10^{6} / \mathrm{ml}, 25 \%$ motility and $60 \%$ abnormal forms. This pregnancy progressed to term.

\section{Discussion}

In this study two micromanipulation techniques utilized in the treatment of male factor infertility (SUZI and PZD) have been compared. The SUZI technique seems to provide patients with oligozoospermia of $<10 \times 10^{6}$ spermatozoa $/ \mathrm{ml}$ with a higher chance of obtaining two PN eggs. Only two of the nine patients with $<10 \times 10^{6}$ spermatozoa/ml achieved a two PN egg by the PZD technique, even though enough spermatozoa were harvested to form an insemination droplet. In patients who had $>10 \times 10^{6}$ spermatozoa $/ \mathrm{ml}$ there was no significant difference between the two techniques.

The quality of spermatozoa recovered in cases of severe oligozoospermia combined with moderate asthenozoospermia and/or teratozoospermia is severely reduced compared to patients with a higher sperm number in the ejaculate. Cohen et al. (1991) have also reported that patients treated by PZD with $0-5 \times 10^{6}$ and $6-10 \times 10^{6}$ spermatozoa/ml in their semen have lower fertilization rates than when $>11 \times 10^{6}$ spermatozoa/ml are present in the semen. In addition to the effect of oligozoospermia,

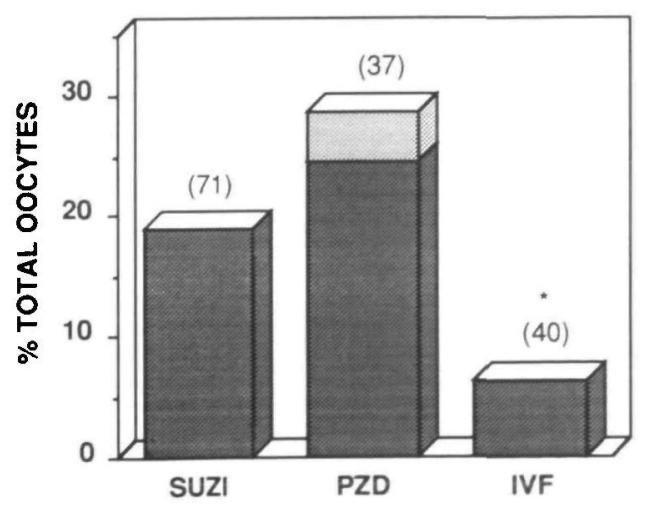

Fig. 3. Comparison of the percentage of two pronuclear (河) and polyspermic (웅) oocytes obtained after performing sub-zonal sperm microinjection (SUZI), partial zona dissection (PZD) and routine in-vitro fertilization (IVF) using spermatozoa from six patients. The number of oocytes treated is in parentheses and originally came from the partners of the six male factor patients, all of whom had $>16$ oocytes collected ( ${ }^{*} P<0.01$ compared to SUZI and PZD). Semen specimens incubated with 2-deoxyadenosine in all cases. 
Cohen et al. (1991) also reported that motility and morphology affected the chance of fertilization.

Although a number of pregnancies have been established using micromanipulation techniques, the major limiting step in treating male factor patients is fertilization. The fertilization rate per oocyte treated in routine IVF using normal semen ranges from 60 to $80 \%$. The fertilization rate when treating male factor infertility cases, however, varies considerably depending on the sperm defects. In less severe cases of male factor infertility where IVF can still be performed, the fertilization rates vary between 30 and $40 \%$. In our initial study, the use of mini-Percoll enabled male factor patients to achieve fertilization while, in contrast, no fertilizations were obtained using the conventional swim-up technique (Table I). Previous studies have shown that the miniPercoll technique allows a greater concentration of spermatozoa to be harvested (Ord et al., 1990; $\mathrm{Ng}$ et al., 1992); however, the quality of spermatozoa recovered does not appear to be correspondingly increased. $\mathrm{Ng}$ et al. (1992) reported that the swim-up procedure produced a preparation with better quality characteristics (velocity, morphology and intact acrosomes) than the mini-Percoll technique. In two studies (Cohen et al., 1989; Malter and Cohen, 1989) comparing IVF and PZD as treatments of male factor infertility, the incidence of monospermic fertilization after IVF was $16 / 30(56 \%)$ and $15 / 45(33 \%)$ respectively, compared to $27 / 34(79 \%)$ and $28 / 50(56 \%)$ respectively when PZD treated oocytes were fertilized. In this trial, patients' sibling oocytes were treated by IVF and the micromanipulation techniques, showing that $\mathrm{PZD}$ was a valid method of treatment in cases where the patient has a mild male factor infertility and enough spermatozoa can be obtained to inseminate. In contrast, SUZI appeared to provide no benefit in mild male factor cases.

Although SUZI is the preferred technique in more severe cases of oligozoospermia, the question remains as to whether SUZI or PZD should be chosen for treatment of less severe cases of male factor infertility. The patients with an initial sperm count of $>10 \times 10^{6}$ spermatozoa/ml in this study had an overall monospermic fertilization rate that did not differ when comparing the two techniques. PZD, however, as distinct from SUZI, does not place the onus on the operator to select spermatozoa. PZD allows natural sperm selection to occur so that the fittest spermatozoa will be able to reach the zona pellucida incisions and enter the perivitelline space to fertilize the eggs. Enabling the best spermatozoa to fertilize the eggs may enhance embryo viability as embryos produced after fertilization by male factor spermatozoa may have a lower overall potential than embryos resulting from normozoospermic patients.

With respect to sperm morphology, Oehninger et al. (1988) reported that patients with poor sperm morphology had a lower ongoing pregnancy rate per cycle than patients with normal sperm morphology. Published pregnancy results after SUZI indicate that the rate of biochemical pregnancies and miscarriages may be higher than that occurring when treating patients with normal semen by IVF (Fishel et al., 1991; Palermo et al., 1992; Sakkas et al., 1992). In these three studies a total of four preclinical and two abortions were obtained from 19 pregnancies. Given the low number of cases, it remains to be seen whether this trend will persist as more pregnancies are obtained using the SUZI technique.

This study has shown that fertilization can be improved by the use of the mini-Percoll technique by increasing the number of spermatozoa recovered from an ejaculate; this subsequently increases the quality of spermatozoa in the insemination droplet. In addition when using PZD, a higher number of spermatozoa in the patient's ejaculate also increased the quality of spermatozoa in the insemination droplet. The baseline semen parameters used by us for the use of PZD to treat males exhibiting moderate oligozoospermia are $10-20 \times 10^{6}$ spermatozoa $/ \mathrm{ml}$, motility $>20 \%$ and normal morphology $>40 \%$. Patients exhibiting semen parameters more severe than the above values have a higher chance of obtaining fertilized eggs by the SUZI technique.

\section{References}

Cohen,J., Edwards,R., Fehilly,C., Fishel,S., Hewitt,J., Purdy,J., Rowland,G., Steptoe,P. and Webster,J. (1985) In vitro fertilization: a treatment of male infertility. Fertil. Steril., 43, 422-433.

Cohen,J., Malter,H., Wright,G., Kort,H., Massey,J. and Mitchell,D. (1989) Partial zona dissection on human oocytes when failure of zona pellucida penetration is anticipated. Hum. Reprod., 4, 435-442.

Cohen,J., Talansky,B.E., Malter,H., Alikani,M., Adler,A., Reing,A., Berkely,A., Graf,M., Davis,O., Liu,H., Bedford,J.M. and Rosenwaks,Z. (1991) Microsurgical fertilization and teratozoospermia. Hum. Reprod., 6, 118-123.

Fishel,S., Antinori,S., Jackson,P., Johnson,J. and Rinaldi,L. (1991) Presentation of six pregnancies established by sub-zonal insemination (SUZI). Hum. Reprod., 6, 124-130.

Fishel,S., Jackson,P., Antinori,S., Johnson,J., Grossi,S. and Versaci,C. (1990) Subzonal insemination for the alleviation of infertility. Fertil. Steril., 54, 828-835.

Gianaroli,L., Serachioli,R., Ferraretti,A.P., Trounson,A.O., Flamigni,C. and Bovicelli,L. (1986) The successful use of human amniotic fluid for mouse embryo culture and human in vitro fertilization, embryo culture and embryo transfer. Fertil. Steril., 46, 907-913.

Malter,H.E. and Cohen,J. (1989) Partial zona dissection of the human oocyte: a non traumatic method using micromanipulation to assist zona pellucida penetration. Fertil. Steril., 51, 139-148.

Ng,S.C., Bongso,A., Ratnam,S.S., Sathananthan,H., Chan,C.L.K., Wong,P.C., Hagglund,L., Anandakumar,C., Wong,Y.C. and Goh,V.H.H. (1988) Pregnancy after transfer of multiple sperm under the zona. Lancet, ii, 790.

Ng,F.L.H., Liu,D.Y. and Baker,H.W.G. (1992) Comparison of Percoll, mini-Percoll and swim-up methods for sperm preparation from abnormal sperm samples. Hum. Reprod., 7, 261-266.

Oehninger,M.D., Acosta,A.A., Morshedi,M., Veeck,L., Swnson,R.J., Simmons,K. and Rosenwaks,Z. (1988) Corrective measures and pregnancy outcome in in vitro fertilization in patients with severe sperm morphology abnormalities. Fertil. Steril., 50, 283-287.

Ord,T., Patrizio,P., Marello,E., Balmaceda,J.P. and Asch,R.H. (1990) Mini-Percoll: a new method of semen preparation for IVF in severe male factor infertility. Hum. Reprod., 6, 987-989.

Overstreet,J.W., Yanagimachi,R., Katz,D.F., Hayashi,K. and Hanson,F.W. (1980) Penetration of human spermatozoa into the human zona pellucida and the zona-free hamster egg: a study of fertile donors and infertile patients. Fertil. Steril., 27, 815-831.

Palermo,G., Joris,H., Devroey,P. and Van Steirteghem,A.C. (1992) Induction of acrosome reaction in human spermatozoa used for subzonal insemination. Hum. Reprod., 7, 248-254. 
Quinn,P., Kerin,J.F. and Warnes,G.M. (1982) Improved pregnancy rate in human in vitro fertilization with the use of a medium based on the composition of human tubal fluid. Fertil. Steril., 44, 493-498.

Sakkas,D., Lacham,O., Gianaroli,L. and Trounson,A.O. (1992) Subzonal sperm microinjection in cases of severe male factor infertility and repeated in vitro fertilization failure. Fertil. Steril., 57, $1279-1288$.

World Health Organization (1987) Laboratory Manual for the Examination of Human Semen and Semen-Cervical Mucus Interaction. Cambridge University Press, Cambridge.

Received on August 3, 1992; accepted on December 9, 1992 